\title{
Klinische Überlegungen bezüglich der wundheilungsphasen- adaptierten und gewebespezifischen Dosierung in der Manuellen Therapie
}

\section{Clinical Considerations in the Selection of Manual Therapy Dosage Concerning Adaption to Wound Healing Phases and Specific Soft Tissues}

\author{
Autoren \\ Thomas Koller \\ Institute \\ Rehabilitationsklinik Bellikon, Orthopädische und \\ Handchirurgische Rehabilitation, Bellikon, Schweiz \\ Schlüsselwörter \\ Wundheilung, Bindegewebewiderstand, Dosierung, \\ Mechanotransduktion \\ Key words \\ wound healing, soft tissue resistance, dosage, \\ mechanotransduction \\ eingereicht 12.9 .2018 \\ akzeptiert 17.9.2018 \\ Bibliografie \\ DOI https://doi.org/10.1055/a-0822-1722 \\ manuelletherapie 2019; 23: 40-46 \\ (c) Georg Thieme Verlag KG Stuttgart · New York \\ ISSN 1433-2671
}

\author{
Korrespondenzadresse \\ Thomas Koller \\ Fachexperte OHR Therapien \\ Orthopädische und Handchirurgische Rehabilitation \\ MAS msk ZFH, OMT svomp, Dipl. PT FH \\ Rehaklinik Bellikon \\ Mutschellenstr. 2 \\ 5454 Bellikon \\ Schweiz \\ thomas.koller@rehabellikon.ch
}

\section{ZUSAMMENFASSUNG}

„Gebrauch bestimmt die Funktion“! Für eine funktionelle Ausrichtung (Mechanotransduktion) reichen wahrscheinlich schon minimale Kräfte von außen aus. Der zweite Bindegewebewiderstand (R2) scheint klinisch ein guter Anhaltspunkt für die adäquate Dosierung in der Proliferationsphase zu sein und somit einer potenziellen Überdosierung entgegenzuwirken.

\section{ABSTRACT \\ “Usage determines function”! Probably even minimal exterior forces may be sufficient for functional alignment (mechano- transduction). The second soft tissue resistance (R2) seems to be a good indicator for adequate dosage during the prolifer- ation phase and assets the avoidance of possible overdosage.}

\section{Einleitung}

Das Ziel der physiotherapeutischen Behandlung nach einem Trauma oder einer Operation besteht stets darin, die Funktion auf Struktur- und Aktivitätsebene wiederzuerlangen. Dies gilt als Voraussetzung und Vorbereitung, um den Übertrag auf die Partizipationsebene zu ermöglichen und oder erleichtern. Grundvoraussetzung dafür ist das Wiedererlangen der funktionellen Integrität auf Gewebeebene, was vornehmlich durch eine Operation oder Ruhigstellung der betroffenen Strukturen (Gewebe) geschieht.
In der physiotherapeutischen Nachbehandlung werden sogenannte funktionelle Reize gesetzt, mit der Absicht, diese betroffene Struktur auf Zellebene einschließlich der extrazellulären Matrix positiv zu beeinflussen. Lassen nach einem Trauma oder einer Operation die orthopädischen Limits eine funktionelle Reizgebung zu, besteht das Verständnis der funktionellen Ausrichtung der betroffenen Gewebe analog der bekannten Gewebephysiologie und Wundheilungsphysiologie [1][2]. Kann durch einen Fixateur externe oder eine zirkuläre Gipsanlage aber nur bedingt oder gar nicht 
funktionell gereizt werden, stellt sich unweigerlich die Frage: Wie viel funktioneller (mechanischer) Reizgebung bedarf es, damit auf Zellebene (also im Gewebe) Vorgänge in Aktion treten, welche das Gewebe auf den äußeren Reiz adaptieren lassen?“. Bekannt ist, wenn keine mechanischen Reize von außen appliziert werden keine funktionelle Ausrichtung stattfindet und zu viele mechanische Reize von außen wirken - das Gewebe mit einer Überlastungsreaktion (Zellschaden und erneute Entzündungsreaktion) reagiert [3][4][5][6][7].

Aber welche Dosierung ist während der Wundheilungsphasen in Bezug auf eine funktionelle Reizgebung auf Zellebene adäquat? Im Folgenden werden verschiedene diesbezügliche Aspekte (Puzzleteile) erläutert und in einer „klinischen Schlussfolgerung“ zusammengefasst.

\section{Mechanotransduktion}

Mechanotransduktion bedeutet die Reaktion im Innern einer Zelle auf einen mechanisch applizierten Reiz von außen. Bei adäquater Reizung reagiert die Zelle mit einer Gentranskription und beeinflusst somit die intra- und extrazelluläre Matrix und schließlich die Trophik (Qualität) des betroffenen Gewebes [3][4][5][8][9][10].

Bouffard et al. [5] beobachteten nach einer Gewebeverletzung durch eine 20-30\%ige Gewebedehnung während täglich 10 Minuten einen verminderten Anstieg sowohl des Transforming Growth Factor Beta 1 (TGF-Beta-1) als auch des Typ-1-Prokollagen. Bei TGFBeta- 1 handelt es sich um ein lokales Zytokin, das im Zusammenhang mit der Wundheilung und der Fibrosierung von Gewebe förderlich wirkt. Eine zu hohe Aktivität von TGF-Beta- 1 führt zu einer überdurchschnittlichen Fibrosierung und somit einer tendenziellen Restriktion von Narbengewebe [11][12][13]. Bouffard et al. [5] sehen in der Gewebedehnung während der Wundheilung durch diese „mechanische“ Hemmung einen positiven Effekt auf eine verminderte Kollagensynthese.

Balestrini und Billar [4] wiesen bei täglicher $5 \%$ iger Gewebedehnung über jeweils ein paar Stunden einen positiven Effekt auf die Dehnfähigkeit der extrazellulären Matrix nach. Sie fanden aber auch heraus, dass ein zu starker und zu lange andauernder Dehnreiz zu einer vermehrten Kollagensynthese und damit zu einer vermehrten Restriktion von Gewebe führte [4].

In ihrem Review fassten Andalib et al. [3] selektionierte Studien zur Mechanotransduktion zusammen. Die Autoren gaben unter anderem auch die verwendeten mechanischen Testkräfte auf die Zellen (in der alten Maßeinheit „Dyne“, die seit 1978 durch die SIEinheit „Newton“ ersetzt wurde). Die Umrechnung ergab, dass jede Zelle mit einer Kraft von 0,00002-0,00058 N mechanisch stimuliert wurde und alle in den Review eingeschlossenen Studien einen mechanotransduktorischen Effekt nachweisen konnten [3].

Diese Erkenntnissen verdeutlichen, dass Zellen sehr mechanosensitiv sind und eine Zellreaktion auf einen mechanischen Stimulus nur minimale Kräfte erfordert.

In > Abb. 1 ist der mechanotransduktorische Vorgang schematisch dargestellt. Ein mechanischer Stimulus von außen stimuliert die Mechanosensoren der Zellmembranen (hier am Beispiel eines Fibroblasten). Diese aktivieren wiederum sogenannte „Adapterproteine“, welche sich auf der Zellkernmembran (Nuklearmembran) befinden. Die Adapterproteine leiten den mechanischen Reiz auf das Zytoskelett und stimulieren die Skelettstruktur so, dass die Zelle mit einer Gentranskription reagiert und somit veränderte (angepasste) Vorgänge und Produkte an die intra- und extrazelluläre Matrix weitergibt. Damit ist eine Zellantwort auf einen mechanischen Reiz erfolgt [5][14].

\section{Funktionelle Ausrichtung}

Der Begriff „funktionelle Ausrichtung“ beschreibt die gebrauchsadaptierte Ausrichtung der extrazellulären Bestandteile wie Kollagen, Elastin etc. durch die Mechanotransduktionseigenschaften jeder einzelnen Zelle. Je früher nach einem Trauma oder einer Operation diese funktionelle Reizsetzung erfolgt, desto weniger Umbauprozesse finden in der Remodulierungsphase statt. Grund dafür ist, dass sich der unspezifische Kollagen-Typ-III während der Proliferationsphase bei adäquatem mechanischen Stimulus funktionell ausrichtet [1]. Wird der unspezifische Kollagen-Typ-III in der Remodulierungsphase allmählich vom spezifischen Kollagentyp-I und Kollagentyp-II ersetzt, wäre eine schon funktionelle Ausrichtung des Kollagen-Typ-III von Vorteil.

In der Remodulierungsphase werden die definitiven KollagenTypen jeweils in gleicher Ausrichtung wie der unspezifische Kollagen-Typ-III angeordnet. Beim nachfolgenden Belastungs- oder Trainingsaufbau resultiert die bereits funktionelle Ausrichtung in einer weniger ausgeprägten Umbauphase. Bei einem nicht funktionell ausgerichteten posttraumatisch oder operativ versorgten Gewebe finden die Umbauprozesse viel intensiver statt. Ein Umbau- oder Adaptionsprozess auf Training oder mehr Belastung von Gewebe geht immer mit einer temporären Gewebeschwächung einher. Dieser intensive Umbauprozess stellt gegen Ende der Remodulierungsphase ein erhöhtes Risiko für eine Wiederbeschädigung dar. Dies gilt es in der Rehabilitation stets zu berücksichtigen.

- Abb. 2 erläutert die kollagene Ausrichtung und die mechanische Reizsetzung bezüglich Dosierung. Wird nicht mechanisch gereizt, fehlt die funktionelle Ausrichtung, wird zu viel gereizt, entsteht eine potenzielle Zellschädigung mit einer erneuten Entzündungsreaktion [2][6]. Hier ist zu vermerken, dass es natürlich Dysfunktionen gibt, bei denen therapeutisch bewusst und gezielt eine Verletzung mit einer mechanischen Überdosierung erreicht werden soll. In diesem Zusammenhang wären Triggerpunkt-Behandlungen und das Lösen von Gewebe- und Kapselverklebungen im Sinne einer Beweglichkeitssteigerung zu erwähnen.

\section{Wundheilungs- und Turn-over-Zeiten spezifischer Gewebe}

Nicht jedes Gewebe reagiert mit einer gleich heftigen und gleich langen Entzündungsreaktion [15]. Einige Gewebearten zeigen sogar praktisch keine Entzündungsreaktionen, wie z. B. solche, die nur über Diffusion vital erhalten werden. Ein klassisches Beispiel ist das intrinsische Sehnengewebe, welches vornehmlich mit der Achillodynie in Verbindung steht. Allgemein gilt, dass gut durchblutetes Gewebe gegenüber weniger gut vaskularisiertem Gewebe eine heftigere Entzündungsreaktion und kürzere Wundheilungsphasen aufweist. 


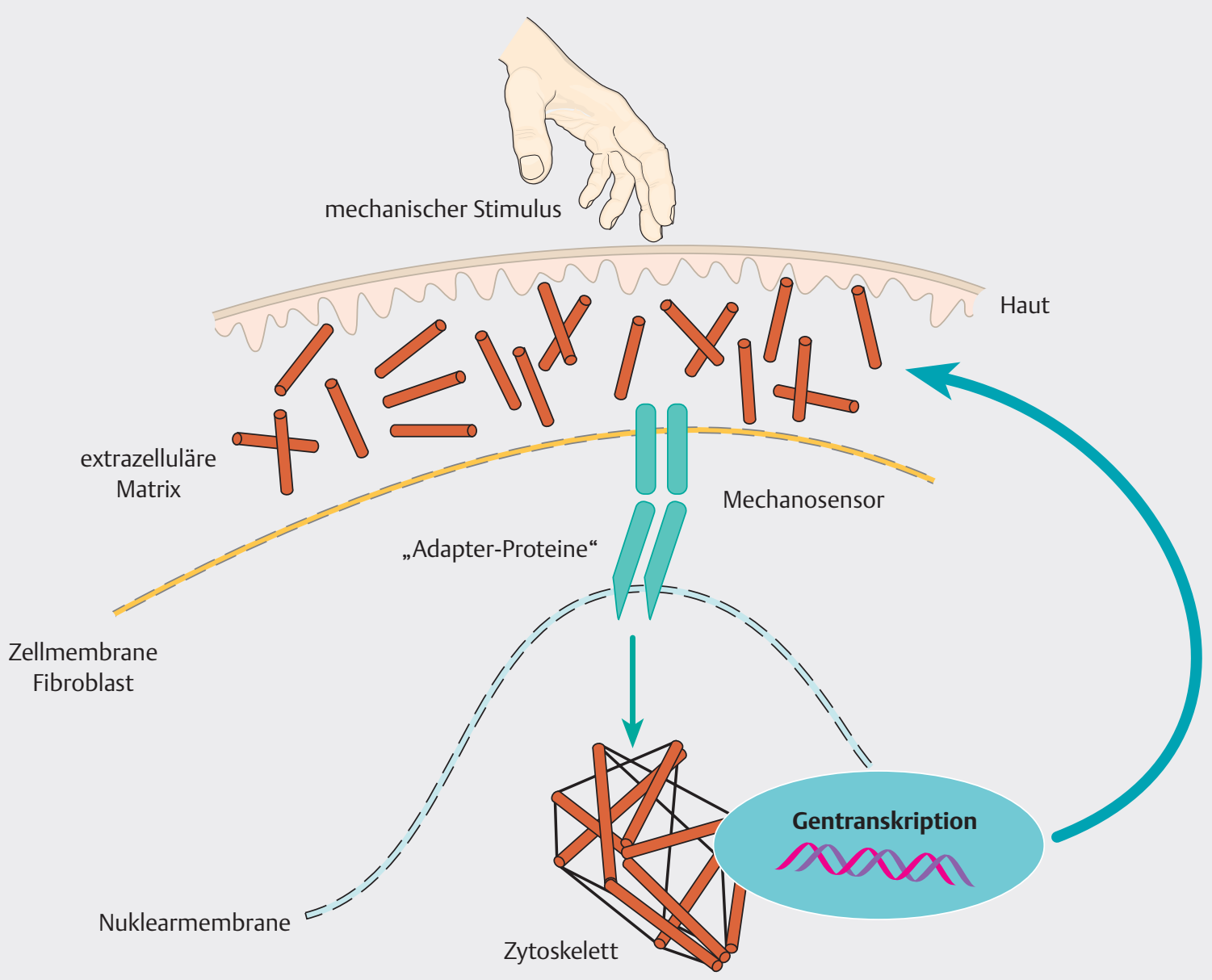

Abb. 1 Vereinfachte Darstellung der Mechanotransduktion am Beispiel einer Fibroblastenzelle (adaptiert nach Bouffard et al. [5]). (Quelle: T. Koller; graf. Umsetzung: Thieme Gruppe)

- Tab. 1 Wundheilungszeiten spezifischer Gewebe nach de Morree [15]. Während der Proliferationsphase sollte manuell vorsichtiger als in der Remodulierungsphase dosiert werden. (Quelle: Koller T. Physiotherapeutische Diagnostik. Hypothesengeleitet und klinisch relevant entscheiden. Stuttgart: Thieme; 2017)

\begin{tabular}{|c|c|c|c|}
\hline & Entzündungsphase & Proliferationsphase & Remodulierungsphase \\
\hline Kapsel-Bindegewebe & 0. bis 3. Tag bis 5. Tag & 3. bis 5 . Tag bis 6 . Woche & ab 6. Woche \\
\hline Meniskus & 0 . bis 5. Tag & 5. Tag bis 10 . Woche & ab 10. Woche \\
\hline Discus intervertebralis & 0. bis 5 . Tag & 5. Tag bis 3 . Woche & ab 3. Woche \\
\hline $\begin{array}{l}\text { Sehnengewebe: } \\
\text { extrinsisch } \\
\text { intrinsisch }\end{array}$ & $\begin{array}{l}\text { 0. bis } 3 . \text { Tag bis } 5 . \text { Tag } \\
\text { nur bedingt }\end{array}$ & $\begin{array}{l}\text { 3. bis } 5 \text {. Tag bis } 4 \text {. Woche } \\
\text { 9. bis } 12 \text {. Woche }\end{array}$ & $\begin{array}{l}\text { ab } 4 \text {. Woche } \\
\text { ab 9. bis } 12 \text {. Woche }\end{array}$ \\
\hline Knochen & 0. bis 3. Tag bis 5 . Tag & 3. bis 5. Tag bis 6 . Woche & ab 4 . Woche bis 8 . bis 12 . Woche \\
\hline Sehnen-Knochen-Übergang & 0. bis 5. Tag & 5. Tag bis 6 . Woche & ab 4. bis 6 . Woche \\
\hline Muskelgewebe & 0. bis 4. Tag & 4. Tag bis 3. Woche & ab 3. Woche \\
\hline
\end{tabular}

In $>$ Tab. 1 sind die verschiedenen Wundheilungszeiten nach de Morree [15] gegenübergestellt. Für die manuelle Dosierung ergibt sich daraus folgende Konsequenz: Je länger die Proliferationsphase des betroffenen Gewebes dauert, desto länger ist auch der unspezifische und provisorische Kollagen-Typ-III im Gewebe aktiv. Daher sollte auch länger vorsichtiger manuell dosiert werden [2].
Die Turn-over-Zeiten beschreiben den vollständigen Zyklus des Auf- und Abbaus einer Gewebestruktur auf Zellebene (extrazellulärer Raum). In $>$ Tab. 2 ist eine Auswahl von spezifischem Gewebe aufgelistet. Durch ein Trauma oder eine Operation wird die Integrität des betroffenen Gewebes zerstört. Zumeist findet danach eine 

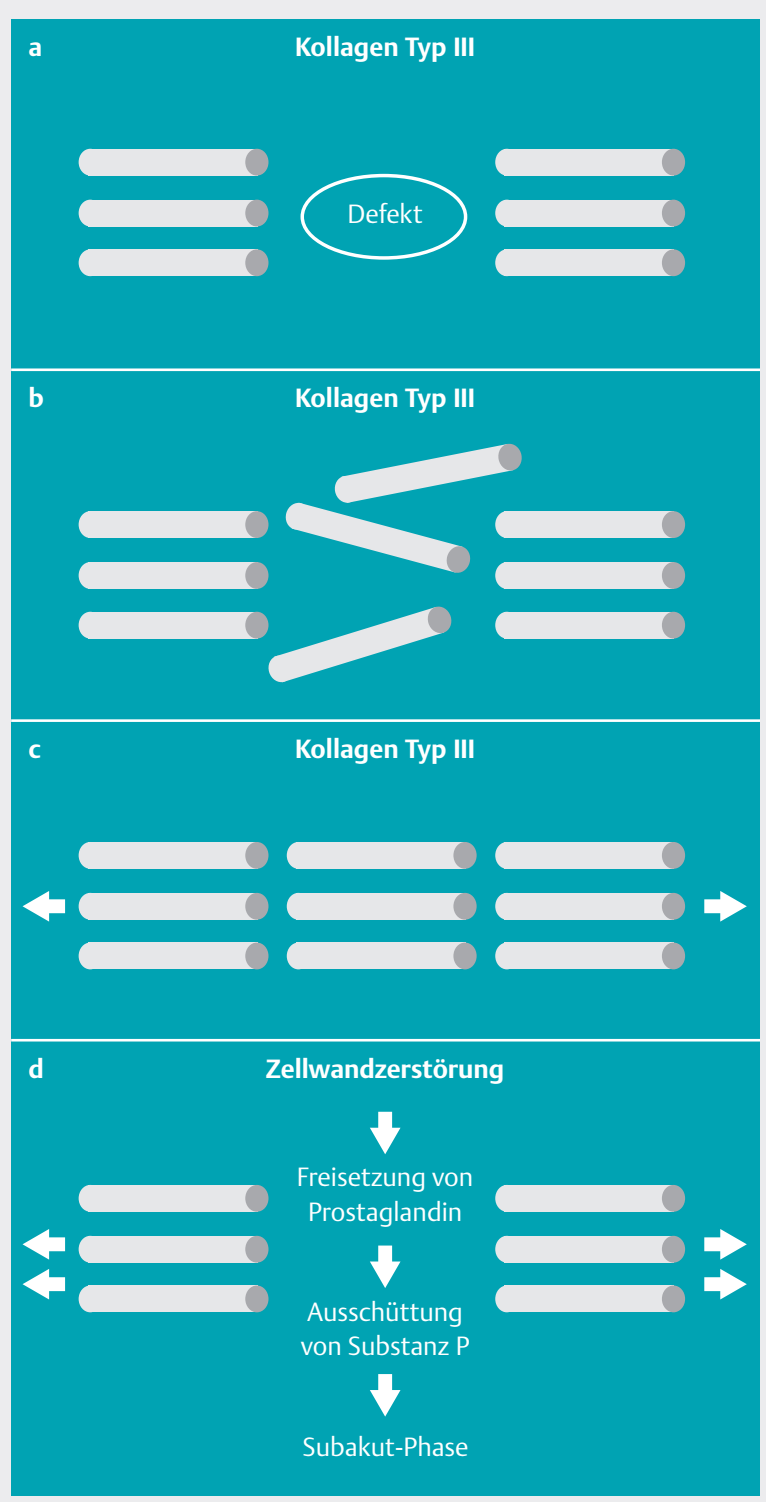

-Abb. 2 Gewebereaktion auf unterschiedlich dosierte mechanische Reize während der Wundheilung. (Quelle: Koller T. Physiotherapeutische Diagnostik. Hypothesengeleitet und klinisch relevant entscheiden. Stuttgart: Thieme; 2017)

gewisse Entlastungsphase statt (Fraktur, Sehnennaht etc.). Nach dieser Entlastungsphase wird wieder sukzessive belastet.

Vor allem bei den Gelenken sollten den Turn-over-Zeiten von Synovialflüssigkeit, Hyaluronsäure und Glykosaminoglykane Rechnung getragen und diese im Belastungsaufbau berücksichtigt werden. Durch die lange Entlastungsphase haben sich die erwähnten Strukturen der „Nichtbelastung“ angepasst. Da sich die Glykosaminoglykanketten vermindert haben, können sie weniger Wasser im Knorpel binden. Dies hat zur Folge, dass der Knorpel „weich“ wird (da der Kollagen-Typ-II immer noch genau gleich vorhanden ist). Wird nun der Belastungsaufbau forciert, besteht die Gefahr, dass die Knorpelstruktur für die Adaptation zu wenig Zeit hat und mit einer Gewebeschädigung reagiert. Erhält der kontinuierliche
- Tab. 2 Turn-over-Zeiten spezifischer Gewebe: ein relevanter Faktor bezüglich Belastungsaufbau und temporärer Gewebeschwächung als Reaktion auf Belastungsänderungen durch neue Aktivitäten (Arbeit, Sport etc.) nach Diemer [16] und van den Berg [17]. (Quelle: Koller T. Physiotherapeutische Diagnostik. Hypothesengeleitet und klinisch relevant entscheiden. Stuttgart: Thieme; 2017)

\begin{tabular}{|l|l|}
\hline Struktur/Gewebe & Turn-over-Zeit \\
\hline $\begin{array}{l}\text { Kollagen-Typ-1 (z. B. Bandstrukturen, } \\
\text { Sehnen etc.) }\end{array}$ & 300 bis 500 Tage \\
\hline \begin{tabular}{l} 
Kollagen-Typ-II (z. B. Knorpelgewebe) \\
\hline $\begin{array}{l}\text { Kollagen-Typ-III (unspezifisch in der } \\
\text { Proliferationsphase) }\end{array}$
\end{tabular} & 30 bis 100 Jahre (im Labor) \\
\hline $\begin{array}{l}\text { Synovialflüssigkeit } \\
\text { Kapselgewebe }\end{array}$ & 9 bis 14 Tage \\
\hline Hyaluronsäure & 14 bis 21 Tage \\
\hline Matrix & 2 bis 4 Tage \\
\hline Glykosaminoglykane & 2 bis 9 Tage \\
\hline Knochengewebe & 7 bis 10 Tage \\
\hline
\end{tabular}

Belastungsaufbau 2 bis 3 Wochen Zeit, ist das Risiko einer Gewebeschädigung deutlich geringer.

Eine weitere Thematik in Bezug auf die Turn-over-Zeiten sind die „New-use“-Aktivitäten. Wird Gewebe durch eine neue Sportart oder ein massiv intensiveres Training stimuliert, beginnt ein Umbauprozess der belasteten Strukturen. Diese Umbauprozesse führen stets zu einer temporären Schwächung der Gewebestruktur. Bei gleichzeitig intensiverem und über eine längere Dauer stetig belastungssteigerndem Training erhöht sich somit das Risiko einer (Wieder-)Verletzung [2].

\section{Direkte und indirekte Schmerzleitung}

\section{Direkte Schmerzleitung}

Physiologisch besitzt jedes Gewebeareal sein eigenes Abbild auf dem Kortex. Demzufolge lassen sich alle als schmerzhaft empfundenen Reize klar lokalisieren, um so eine zeitnahe motorische Schutzantwort zu generieren. Da Patienten theoretisch mehr oder weniger den A-delta-Faserschmerz vom C-Faserschmerz unterscheiden können, sind ihre diesbezüglichen Angaben für die Dosierung der manualtherapeutischen Interventionen äußerst hilfreich. Im Klartext: Bei der körperlichen Untersuchung und der Behandlung dürfen die Patienten bei der entsprechenden Dosierung gerade noch ein „Ziehen“ wahrnehmen (A-delta-Faser-Aktivität). Sie sollten jedoch unter allen Umständen kaum bzw. niemals anhaltende Schmerzen empfinden (C-Faser-Aktivität), da dies ansonsten eine erneute Entzündungsreaktion auslöst ( $\mathbf{A} \mathbf{b b}$. 3; [1][2][18]).

\section{Indirekte Schmerzleitung}

Bei jeder Verletzung von Gewebsstrukturen erfolgt eine indirekte Schmerzleitung. Weil das primäre Wundgebiet wegen der partiellen Zerstörung von Schmerzfasern temporär nicht in der Lage ist, die nozizeptiven Reize direkt an den Kortex weiterzuleiten, kompensiert das sekundäre Wundareal die Schmerzafferenzen. Bis je- 


\section{Keine Kompensation}

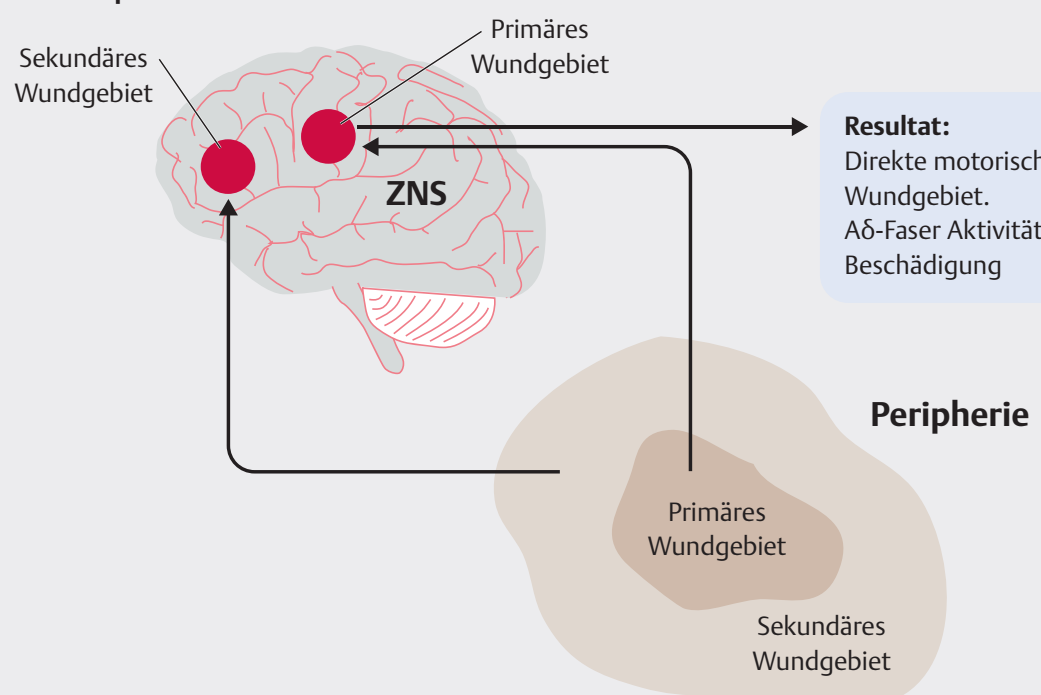

Abb. 3 Direkte Schmerzleitung.(Quelle: Koller T. Physiotherapeutische Diagnostik. Hypothesengeleitet und klinisch relevant entscheiden. Stuttgart: Thieme; 2017)

\section{Kompensation über sekundäres Wundgebiet}

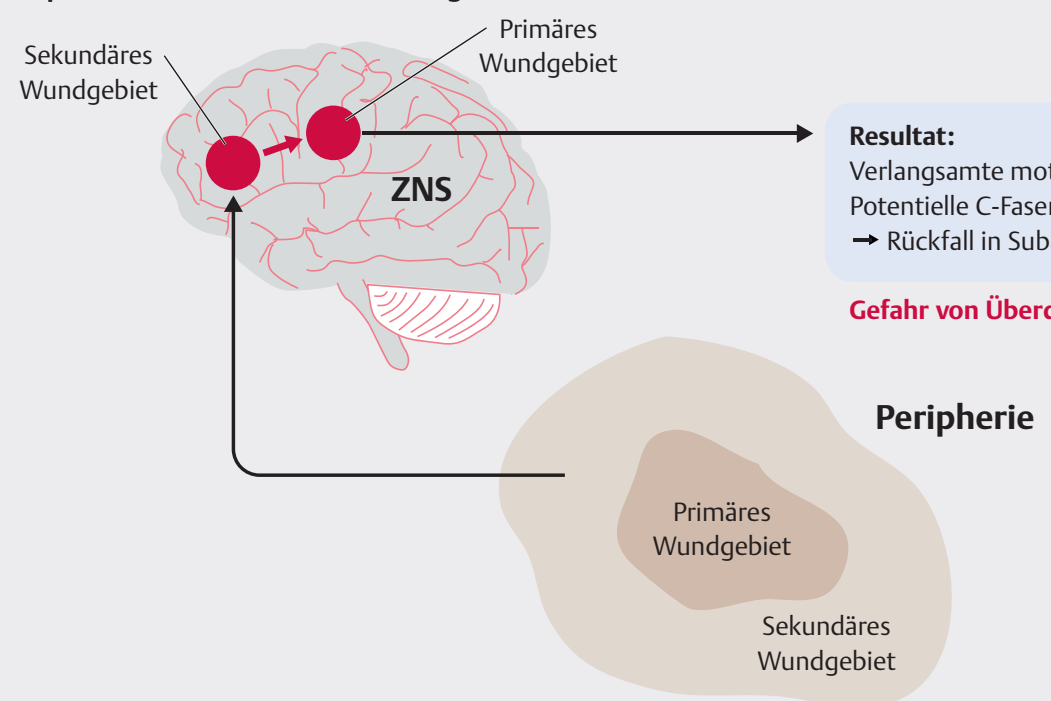

- Abb. 4 Indirekte Schmerzleitung. Quelle: Koller T. Physiotherapeutische Diagnostik. Hypothesengeleitet und klinisch relevant entscheiden. Stuttgart: Thieme; 2017

doch auch das sekundäre Wundgebiet den im primären Wundareal mechanisch gesetzten Reiz wahrnimmt, drohen im primären Wundgebiet bereits Zellwandschädigungen und entsprechend unter dem Aspekt der Wundheilungsphasen - ein Rückschritt in die Entzündungsphase. Die A-delta-Faser ist de facto im primären Wundareal in der Entzündungs- und Proliferationsphase nicht in der Lage, vor einer erneuten zellulären Beschädigung zu warnen ( Abb. 4). Zudem erhält das dem primären Wundgebiet entsprechende kortikale Areal in der Entzündungs- und Proliferationsphase praktisch kaum Afferenzen und ist infolgedessen auf indirekte
Afferenzen aus dem sekundären Wundgebiet angewiesen. Daraus resultieren zum einen die erschwerte differenzierte Wahrnehmung und zum anderen die zusätzlich verlangsamte motorische und verbale Schutzreaktion [1][2][18].

\section{Klinische Schlussfolgerungen}

Die bisherigen Darstellungen lassen keinen klaren Schluss in Bezug auf die absolut richtige Dosierung zu. Das Zusammensetzen der einzelnen Puzzleteile und diesbezügliche Überlegungen ermög- 


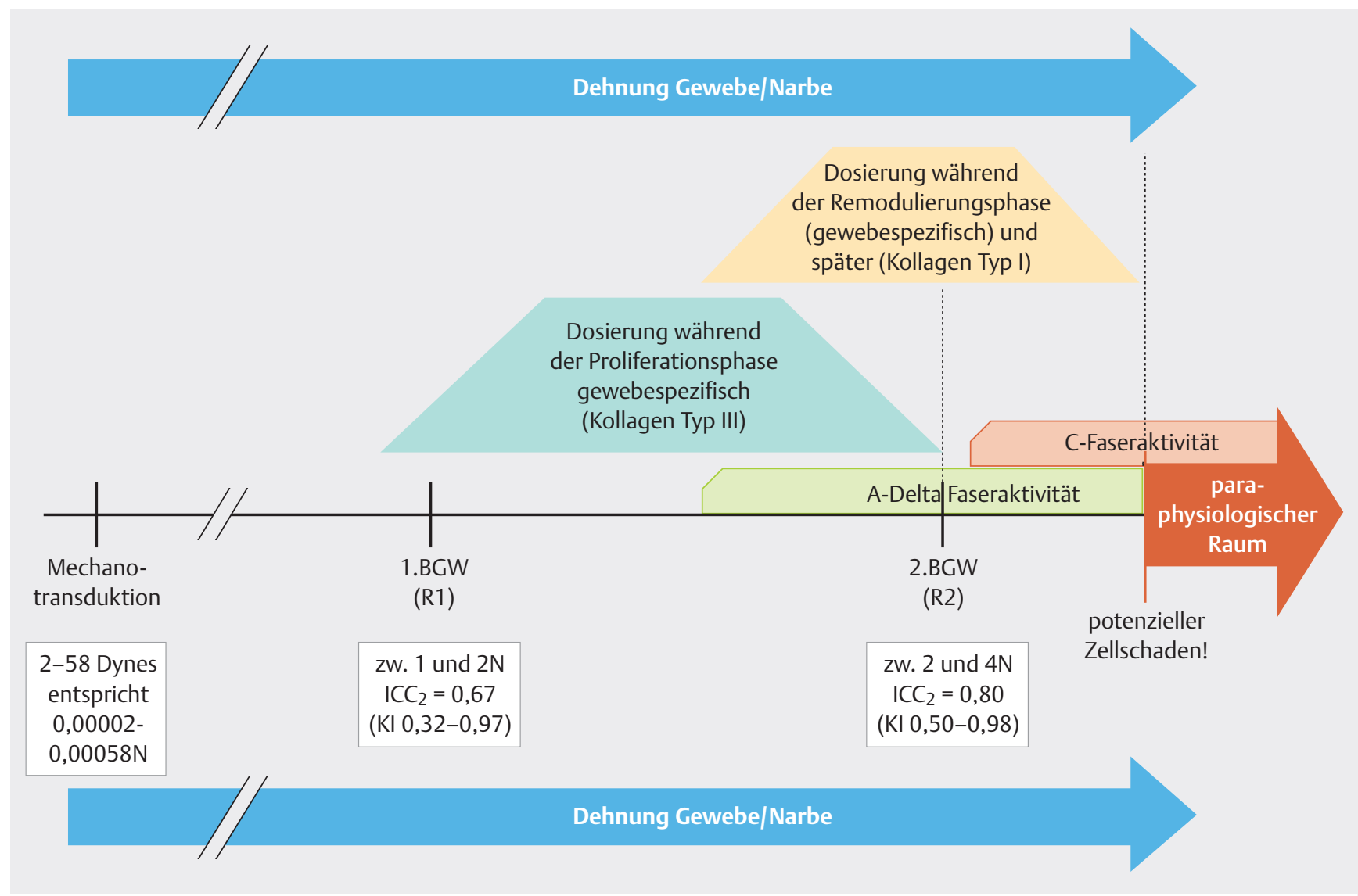

Abb. 5 Zusammenfassende Darstellung der wundheilungsphasenadaptierten und gewebespezifischen Dosierung in der Manuellen Therapie. (Quelle: T. Koller; graf. Umsetzung: Thieme Gruppe)

lichen jedoch das Herantasten an eine adäquate und funktionelle Dosierung.

Die Tatsache, dass schon minimalste Kräfte (bis 0,00058 N) mechanotransduktorische Zellantworten einleiten, zeigt, dass eine funktionelle Ausrichtung wahrscheinlich schon bei sehr sanften manualtherapeutischen Interventionen stattfindet. Die empirisch bekannten Bindegewebewiderstände R1 und R2 liegen (am Rücken) im Bereich von $\mathrm{R} 1$ bei $1-2 \mathrm{~N}$ und im Bereich von R2 bei 2-4 $\mathrm{N}$ [19][20]. Somit ist anzunehmen, dass manuelle Interventionen im Bereich von R1 wahrscheinlich einen ausreichenden Reiz für das Gewebe darstellen, damit eine mechanotransduktorische Antwort der Zelle und eine funktionelle Ausrichtung der extrazellulären Matrix entsteht.

Die Gefahr einer Überdosierung in der Proliferations- und Remodulierungsphase scheint doch höher als keine funktionelle Ausrichtung zu sein. Klinische Erfahrungen vor allem bei schwerstbrandverletzten Patienten zeigten in der Rehaklinik Bellikon und im Universitätsspital Zürich, dass in der Proliferationsphase die manualtherapeutischen Interventionen lediglich im Bereich des ersten Bindegewebewiderstands (R1) deutlich weniger Rückfälle aufgrund von Überbelastungen (Zellschaden) zu beobachten sind. In der gleichen Zeit war auch eine verbesserte Mobilität und Gelenksbeweglichkeit festzustellen.

In der Proliferationsphase scheint R2 (außer bei schwerstbrandverletzten Patienten) ein wichtiger Anhaltspunkt für eine wundheilungsadapierte Dosierung zu sein. In einer Pilotstudie erzielte Koller
[20] bei der Intertester-Reliabilität zur Erkennung von R1 und R2 viel versprechende Resultate. Er erkannte den ersten Bindegewebewiderstand (R1) konnte mit einer moderaten Intertester-Reliabilität $\left(\mathrm{ICC}_{1}=0,67\right)$ und den zweiten Bindegewebewiederstand mit einer guten Intertester-Reliabilität $\left(\mathrm{ICC}_{2}=0,80\right)[20]$.

Für eine adäquate Dosierung in der Proliferationsphase ist wahrscheinlich vor allem die gute Reliabilität von R2 wichtig. In Bezug auf die direkte und indirekte Schmerzleitung ist davon auszugehen, dass die C-Faseraktivität zunehmend im zweiten Bindegewebewiderstand (R2) aktiv werden wird und bis R2 vornehmlich die A-Delta-Faser als „Vorwarner" vor einer Zellschädigung aktiv ist.

Somit lassen sich für die adäquate Dosierung die folgenden klinischen Schlussfolgerungen ziehen ( $\bullet$ Abb. 5):

- Manualtherapeutische und funktionell ausgerichtete Interventionen sollen in der Proliferationsphase bis an den zweiten Bindegewebewiderstand (R2) dosiert werden. Die Dauer der Behandlung mit dieser Dosierung hängt direkt von der Proliferationsphase und den Turn-over-Zeiten der betroffenen Strukturen ab.

- In der gewebespezifischen Remodulierungsphase soll aufgrund funktioneller Reizgebung bezüglich Belastbarkeit und Festigkeit der betroffenen Strukturen im Sinne einer Angleichung an die alltagsrelevanten Aktivitäten einschließlich Sport zunehmend in den zweiten Bindegewebewiderstand (R2) hinein behandelt werden. 
- Zudem sind die Schmerzäußerungen der Patienten zu beachten, welche eine zusätzliche, aber niemals alleinige Hilfe für die aktuelle Dosierung darstellen.

\section{Literatur}

[1] Van den Berg F (Hrsg). Angewandte Physiologie. Bd. 1: Das Bindegewebe des Bewegungsapparates verstehen und beeinflussen. Stuttgart: Thieme; 2011

[2] Koller T. Physiotherapeutische Diagnostik. Hypothesengeleitet und klinisch relevant entscheiden. Stuttgart: Thieme; 2017

[3] Andalib MN, Dzenis Y, Donahue HJ et al. Biomimetic substrate control of cellular mechanotransduction. Biomater Res 2016; 20: 11. doi: 10.1186/s40824-016-0059-1

[4] Balestrini JL, Billiar KL. Magnitude and Duration of Stretch Modulate Fibroblast Remodeling. J Biomech Eng 2009; 131: 051005. doi: 10.1115/1.3049527

[5] Bouffard NA, Cutroneao KR, Badger G] et al. Tissue Stretch Decreases Soluble TCF- $\beta 1$ and Type- 1 Procollagen in Mouse Subcutaneous Connective Tissue: Evidence From Ex Vivo and In Vivo Models. J Cell Physiol 2008; 214: 389-395. doi: 10.1002/jcp.21209

[6] Koller T, Ruegg C, Gut V. Physiologische Grundlagen manueller Mobilisation von Narben und Bindegewebe sowie Dosierung bei Patienten mit großflächigen Brandverletzungen. manuelletherapie 2016; 20: 237-241. doi: 10.1055/s-0042-119245

[7] Koller T, Ruegg C, Gut V. Fachwissen: Physiotherapeutische Werkzeuge zur funktionellen Mobilisation von Narben und Bindegewebe sowie Dosierung bei großsflächigen Narbenplatten. manuelletherapie 2017; 21: 238-243. doi: 10.105/s-0043-121555

[8] Eckes B, Nischt R, Krieg T. Cell-Matrix Interactions in Dermal Repair and Scarring. Fibrogenesis Tissue Repair 2010; 3: 4. doi: 10.1186/17551536-3-4

[9] Huang C, Holfeld J, Schaden W et al. Mechanotherapy: revisiting physical therapy and recruiting mechanobiology for a new era in medicine. Trends Mol Med 2013; 19: 555-564. doi: 10.1016/j. molmed.2013.05.005

[10] Khan KM, Scott A. Mechanotherapy: How physical therapists' prescription of exercise promotes tissue repair. 2009. www.bjsm.bmj. com/content/43/4/247 (12.09.2018)

[11] Kapp H, Smola H. Regulation der Wundheilung durch Wachstumsfaktoren und Zytokine. Hartmann WundForum 2006; 1: 8-14

[12] Lindahl GE, Chambers RC, Papakrivopoulou J et al. Activation of fibroblast procollagen alpha $1(\mathrm{I})$ transcription by mechanical strain is transforming growth factor-beta-dependent and involves increased binding of CCAAT-binding factor (CBF/NF-Y) at the proximal promoter. J Biol Chem 2002; 277: 6153-6161

[13] Wipff PJ, Rifkin DB, Meister J] et al. Myofibroblast contraction activates latent TGF-beta1 from the extracellular matrix. J Cell Biol 2007; 179: 1311-1323

[14] Langevin HM, Bouffard NA, Fox JR. Fibroblast cytoskeletal remodeling contributes to connective tissue tension. J Cell Physiol 2011; 226: 1166-1175. doi: 10.1002/jcp.22442

[15] De Morree J]. Dynamik des menschlichen Bindegewebes: Funktion, Schädigung und Wiederherstellung. München: Urban \& Fischer; 2001

[16] Diemer F, Sutor V. Praxis der medizinischen Trainingstherapie. Stuttgart: Thieme; 2018

[17] Van den Berg F. Angewandte Physiologie Bd. 1: Das Bindegewebe des Bewegungsapparates verstehen und beeinflussen. Stuttgart: Thieme; 2016
[18] Butler D, Moseley GM, Ween-Vermazen G et al. Schmerzen verstehen. Berlin: Springer; 2016

[19] Koller T. Manualtherapeutische Bestimmung der Anstiege des Bindegewebewiderstands bei Narben und Narbenplatten - Pilotstudie. manuelletherapie 2018; 22: 79-85. doi: 10.1055/a-0585-1624

[20] Koller T. Intertesterreliabilität und Kriteriumsvalidität bei der Bestimmung der Haut- und Bindegewebewidestände (BGW) im physiologischen Gewebe - Pilotstudie. manuelletherapie 2019 [zur Publikation angenommen] 